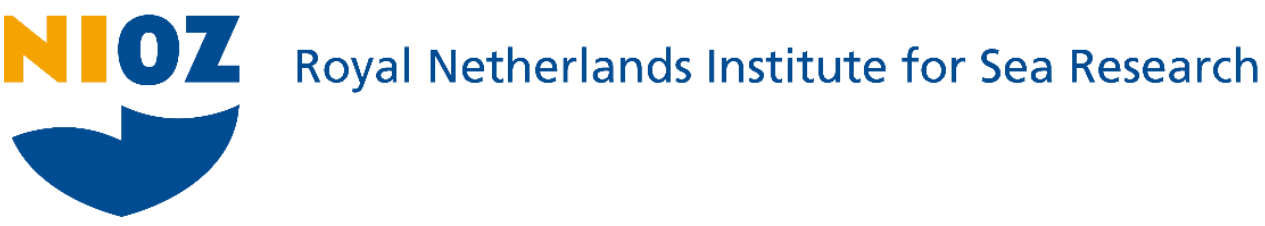

This is a postprint of:

Lyashevska, O., Brus, D.J. \& Meer, J. van der (2016). Gridspacing and the quality of abundance maps for species that show spatial autocorrelation and zero-inflation. Spatial Statistics, 18 (Part B), 386-395

Published version: $\underline{\text { dx.doi.org/10.1016/j.spasta.2016.08.001 }}$

Link NIOZ Repository: $\underline{w w w . v l i z . b e / n l / i m i s ? m o d u l e=r e f \& r e f i d=281997 ~}$

[Article begins on next page]

The NIOZ Repository gives free access to the digital collection of the work of the Royal Netherlands Institute for Sea Research. This archive is managed according to the principles of the Open Access Movement, and the Open Archive Initiative. Each publication should be cited to its original source - please use the reference as presented.

When using parts of, or whole publications in your own work, permission from the author(s) or copyright holder(s) is always needed. 


\title{
Grid-spacing and the quality of abundance maps for species that show spatial autocorrelation and zero-inflation
}

\author{
Olga Lyashevska ${ }^{\mathrm{a}, *}$, Dick J. Brus ${ }^{\mathrm{b}}$, Jaap van der Meer ${ }^{\mathrm{a}}$ \\ ${ }^{a}$ Department of Marine Ecology \\ NIOZ Royal Netherlands Institute for Sea Research \\ P.O. Box 591790 AB Den Burg \\ Texel, The Netherlands \\ ${ }^{b}$ Alterra, Wageningen University and Research Centre \\ P.O. Box 47, 6700AA \\ Wageningen, The Netherlands
}

\begin{abstract}
The effect of grid-spacing on the quality of species abundance maps is explored for species that show zeroinflation and spatial autocorrelation. Using a zero-inflated Poisson mixture model multiple fields of the prevalence parameter $\pi$ and the intensity parameter $\mu$ were simulated. A selected field was sampled by gridsampling with 200,400,800,1600, and $3200 \mathrm{~m}$ grid-spacing and used to predict at a fixed set of validation locations by simple kriging with an external drift. The external drift variables were silt, silt squared and altitude. The estimated sampling distribution of MSE against grid-spacing shows that beyond a spacing of $1600 \mathrm{~m}$ the mean of MSE increases at a much faster rate. Based on these findings the $1600 \mathrm{~m}$ grid which consists of 446 locations for our study area of $2400 \mathrm{~km}^{2}$ gives a compromise between sampling costs and prediction accuracy.
\end{abstract}

Keywords: count data; generalized linear geostatistical modeling; autocorrelation; zero-inflation; grid-spacing

\section{Introduction}

The relationship between species and their environment is generally described by species distribution models; in particular, by habitat suitability or environmental niche models (Guisan and Thuiller, 2005). Such models are constructed using survey data available at a limited set of sampling locations and allow 5 one to create predictive species distribution maps on the basis of environmental data which are usually available for a much larger set of locations (Guisan and Zimmermann, 2000). The number of sampling locations is known to affect the accuracy of the species distribution models and maps (Stockwell and Peterson, 2002; Wisz et al., 2008). Knowledge of the trade-off function between number of sampling locations and accuracy of the predictions is usually not obtained a priori. If number of samples is too low, accuracy will

\footnotetext{
* Corresponding author

Email addresses: olga@herenstraat.nl (Olga Lyashevska), Dick.Brus@wur.nl (Dick J. Brus), Jaap.van.der.Meer@nioz.nl (Jaap van der Meer)
} 
suffer by an unknown ammount: if sampling intensity is too high, the design will be unnecessarily costly. (Caughlan and Oakley, 2001; Reynolds et al., 2011).

Several studies evaluated effects of sample size on the accuracy of species distribution models (Stockwell and Peterson, 2002; Pearson et al., 2007; Wisz et al., 2008; Hanberry et al., 2012). For example, Stockwell and Peterson (2002) assessed sample size requirements for modelling bird species in Mexico by random sampling between 1 and 100 locations. Wisz et al. (2008) considered three sample sizes (10, 30, and 100 locations) to evaluate the quality of model predictions using data for 46 species obtained from natural history collections. Finally, Hanberry et al. (2012) used sample sizes ranging from 30 to 2500 locations to model tree species in northeasten Minnesota. All these studies consider presence-absence maps, but often, predictive species abundance maps in the form of numerical or biomass density are to be preferred, because they are more informative than presence-absence maps (Vieira et al., 2012; Cozzi et al., 2013). Fortin et al. (1989) constructed such maps using sugar-maple tree density data gathered in southwestern Québec. The authors evaluated the ability to predict spatial patterns using different sample sizes and designs. They considered two sample sizes of 50 and 64 points, both derived from a 200-point dataset.

Using real datasets only, as Fortin et al. (1989) did, limits comparison between the effects of different sample designs and sample number sizes as well as uncertainties in the model's parameter values. These limitations were recognised in recent studies, such as those by Perner and Schueler (2004); Rachowicz et al. (2006); Bijleveld et al. (2012) and Foster et al. (2014). An updated approach is to first simulate a spatial field resembling reality as much as possible, a peseudo-reality, which is then subsequently sampled using different sampling designs. The performance of sampling designs is then compared by confronting predictions with simulated values that serve as ground-truth. Zurell et al. (2010) call this the virtual ecologist approach. Following this approach, Biileveld et al. (2012) used the results of an existing intertidal benthic monitoring programme to construct various spatial models with an exponential spatial autocorrelation function. With these models they simulated virtual populations with a Normal distribution and sampled these populations using different sampling designs. They provided a trade-off function between sampling distance and prediction error which was rather flat for those virtual species that hardly showed spatial autocorrelation, but much steeper for species with strong spatial autocorrelation. The assumed normality of the data was clearly violated by the empirical data because of the many zero observations.

The assumption of normality is a common practice, because when dealing with species abundance (count) data the more obvious Poisson distribution is rarely applicable. Ecological count data have two properties that ask for a specific treatment, other than relying on the classical assumption of independent and normally distributed data. These properties are zero-inflation (Martin et al., 2005; Clarke and Green, 1988; Lewis et al., 2011) and spatial autocorrelation, i.e. nearby observations are more similar than observations far apart, even when environmental conditions do not differ. Hitherto most studies have dealt with these two properties, but only one at a time (Tyre et al., 2003; Biileveld et al., 2012).

45 For example, the first property was accounted by Tyre et al. (2003) who considered the zero-inflated 
negative binomial model, but ignored autocorrelation. Ignoring spatial autocorrelation in simulation studies on how sampling designs affect the accuracy of estimates of population- or model parameters or the accuracy of spatial predictions, may lead to biased estimates of this accuracy (Legendre et al., 2002).

Contrary to Tyre et al. (2003), the Bijleveld et al. (2012) study took account of the autocorrelation by

50 simulated normally distributed data. Clearly, there is a need to integrate both properties in a single study and to examine how zero-inflation and autocorrelation may affect recommendations for the optimal sampling design, sample size, and distance between samples.

Studies that simultaneously address zero-inflation and autocorrelation for species abundances (see e.g.

Recta et al., 2012; Boyd et al., 2015) do not treat the question of optimal sampling design. We attempt to fill this gap by following a paper by Lyashevska et al. (2016) in simulating fields with zero-inflated, spatially autocorrelated count data, and sampling the fields repeatedly with different sampling designs. More specifically, we will sample the fields by grid-sampling with a varying spacing. The aim of this paper is to quantify the trade-off between grid-spacing and accuracy of predictions of species-abundance model parameters on a fine grid for mapping, for species that show zero-inflation and spatial aut
these two properties (see Martin et al., 2005, and references therein).

\section{Materials and methods}

\subsection{Data}

Data used in this paper were zero-inflated (66\% are zeros) and autocorrelated counts of a benthic species Macoma balthica (Fig. 1a) that were collected in the yearly Synoptic Intertidal Benthic Surveys (SIBES) monitoring programme conducted in the Dutch Wadden Sea (Biileveld et al., 2012; Compton et al., 2013). The study area, bordered by the barrier islands on the north and by the mainland coast on the south, is formed by intertidal and subtidal mudflats and gullies. The monitoring network consists of 3451 permanent locations on intertidal mudflats at the nodes of a $500 \mathrm{~m}$ grid. The square grid is supplemented by 578 locations. These locations were selected by first selecting 578 out of the 3451 grid-points by simple random sampling without replacement. Then at each selected grid-point one point was selected at a uniformly distributed distance between 0 and $250 \mathrm{~m}$ distance from the grid-point, in a direction randomly chosen from the four directions defined by the grid-lines (Bijleveld et al., 2012). The total sample size was 4029 locations.

The most important determinants of habitat structure used for mapping the abundance were sediment 75 texture characteristic, more specifically the mass fraction of silt, and altitude (Amsterdam Ordnance Datum, Rijkswaterstaat 1). To be used as a predictor in mapping, the covariate must be known everywhere in the study area. Therefore the mass fraction of silt was interpolated by inverse distance weighting in ArcGIS 10.0.

\footnotetext{
${ }^{1}$ www.rijkswaterstaat.nl
} 


\subsection{Overview of evaluation method}

The starting point of the our procedure for evaluating the sampling designs is a model for the spatial distribution of the zero-inflated and autocorrelated count data. This spatial distribution is modelled through a spatial zero-inflated Poisson mixture model(ZIP)(Lambert, 1992; Agarwal et al., 2002):

$$
P\left(Y_{i}=y \mid \eta_{i}\right)= \begin{cases}\pi_{i}+\left(1-\pi_{i}\right) \exp \left(-\mu_{i}\right) & y=0 \\ \left(1-\pi_{i}\right) \frac{\exp \left(-\mu_{i}\right) \mu_{i}^{y}}{y !} & y=1,2,3, \ldots\end{cases}
$$

where $Y_{i}$ is the count at location $i, \eta_{i}$ is spatially dependent random effect, $\pi_{i}$ the probability of a Bernoulli zero at location $i$, and $1-\pi_{i}$ is the probability of a Poisson count, either zero or non-zero. The intensity (mean number of individuals) of the Poisson process at location $i$ is $\mu_{i}$. The first part of the model is the overall probability of zero (Hilbe and Greene, 2007).

The parameters $\pi_{i}$ and $\mu_{i}$ at location $i$ are random variables modelled by the following submodels:

$$
\begin{aligned}
\operatorname{logit}\left(\pi_{i}\right)=\log \left(\frac{\pi_{i}}{1-\pi_{i}}\right) & =\mathbf{x}_{\mathrm{B}, i}^{T} \boldsymbol{\beta}_{\mathrm{B}}+\eta_{\mathrm{B}, i} \\
\log \left(\mu_{i}\right) & =\mathbf{x}_{\mathrm{P}, i}^{T} \boldsymbol{\beta}_{\mathrm{P}}+\eta_{\mathrm{P}, i}
\end{aligned}
$$

with $\mathbf{x}_{\mathrm{B}, i}$ and $\mathbf{x}_{\mathrm{P}, i}$ vectors with covariates at location $i, \boldsymbol{\beta}_{\mathrm{B}}$ and $\boldsymbol{\beta}_{\mathrm{P}}$ vectors with regression coefficients, and $\eta_{\mathrm{B}, i}, \eta_{\mathrm{P}, i}$ residuals of the spatial trend. Note that the model parameters can be modelled by different sets of covariates.

The residuals $\eta_{\mathrm{B}, i}, \eta_{\mathrm{P}, i}$ at any location $i$ are random variables. The probability distribution of the residuals at all locations in the study area was modelled as

$$
\left[\begin{array}{l}
\boldsymbol{\eta}_{\mathrm{B}} \\
\boldsymbol{\eta}_{\mathrm{P}}
\end{array}\right] \sim \mathcal{N}\left(\left[\begin{array}{l}
\mathbf{0} \\
\mathbf{0}
\end{array}\right],\left[\begin{array}{cc}
\mathbf{C}_{\mathrm{B}} & \mathbf{0} \\
\mathbf{0} & \mathbf{C}_{\mathrm{P}}
\end{array}\right]\right)
$$

with $\mathbf{C}_{\mathrm{B}}$ and $\mathbf{C}_{\mathrm{P}}$ covariance matrices. So note that we assumed that the Bernoulli and Poisson residuals were independent. Testing for this assumption we revealed a weak correlation of 0.3. For both random residuals we further assumed isotropy, so that the covariance of the residuals at any two locations was modelled as a function of the distance $h$ between the two locations. For instance, for the Bernoulli residuals, the covariance was modelled as

$$
C_{\mathrm{B}}(h)=\sigma_{\mathrm{B}}^{2} \rho_{\mathrm{B}}\left(h ; \phi_{\mathrm{B}}\right)+\tau_{\mathrm{B}}^{2}
$$

with $\sigma_{\mathrm{B}}^{2}$ the partial sill, $\phi_{\mathrm{B}}$ the range (distance parameter), $\tau_{\mathrm{B}}^{2}$ the nugget, and $\rho_{\mathrm{B}}$ the correlation function, for instance exponential or spherical (Webster and Oliver, 2007).

The two submodels in 2 are generalised linear mixed models, as they are the sum of a linear combination of covariates describing a spatial trend (fixed effect) and a spatially autocorrelated residual (random effect). Diggle (2007) names this type of models as generalised linear geostatistical models. 
Following Diggle (2007), the sum of the trend and residual, representing the transformed model parameter, 95 is referred to as the signal $S$, for instance $S_{\mathrm{B}, i}=\mathbf{x}_{\mathrm{B}, i}^{T} \boldsymbol{\beta}_{\mathrm{B}}+\eta_{\mathrm{B}, i}$. For convenience, all the parameters in one model, including the type of correlation function, are collected in a vector: $\boldsymbol{\theta}_{\mathrm{B}}=\left(\boldsymbol{\beta}_{\mathrm{B}}, \phi_{\mathrm{B}}, \tau_{\mathrm{B}}^{2}, \sigma_{\mathrm{B}}^{2}, \rho_{\mathrm{B}}\right)$ and $\boldsymbol{\theta}_{\mathrm{P}}=\left(\boldsymbol{\beta}_{\mathrm{P}}, \phi_{\mathrm{P}}, \tau_{\mathrm{P}}^{2}, \sigma_{\mathrm{P}}^{2}, \rho_{\mathrm{P}}\right)$. To avoid confusion the model parameters $\boldsymbol{\theta}_{\mathrm{B}}$ and $\boldsymbol{\theta}_{\mathrm{P}}$ are referred to as hyperparameters; with model parameters we mean the parameters $\pi$ and $\mu$.

The aim of evaluating the sampling strategy is to map the prevalence parameter $\pi$ of the Bernoulli distribution and the intensity parameter $\mu$ of the Poisson distribution. Please note that the objective is not to predict the species abundance counts, but to use the observed counts in the sample to estimate, at the desired sites $i$, the expected counts conditional on the values $\mathbf{x}_{\mathbf{i}}$ of the covariates and the random effects $\eta_{i}$ that express spatial dependence. We believe that predicting the counts themselves is not feasible in our situation, and not of practical relevance.

Our evaluation procedure is as follows. The SIBES data are used to estimate the parameters of a ZIP model. Several steps are involved in estimation. First, a ZIP model is fitted by maximum likelihood assuming that both residuals $\eta_{\mathrm{B}, i}$ and $\eta_{\mathrm{P}, i}$ are spatially independent. The fitted model parameters are then used to classify a zero count either as a Bernoulli or a Poisson zero, and to construct two datasets: the Bernoulli dataset with zeros (absent) and ones (present), and the Poisson subset, containing the SIBES locations with a one in the Bernoulli dataset, with counts. In the next step these two data sets are used to fit the two submodels for the parameters $\pi_{i}$ and $\mu_{i}$, but now accounting for spatial autocorrelation. This is done by simulating a large sample of signals $S_{\mathrm{B}}$ and $S_{\mathrm{P}}$ at the SIBES locations by Markov chain Monte Carlo (MCMC) using initial estimates of the hyperparameters, followed by Monte Carlo Maximum Likelihood (MCML) estimation of the hyperparameters. The final MCML estimates of the hyperparameters are used to simulate signals $S_{\mathrm{B}}$ and $S_{\mathrm{P}}$ at the SIBES locations conditional on the observations at these locations.

The fitted hyperparameters are then used to simulate the Bernoulli signal $\left(S_{\mathrm{B}}\right)$ and Poisson signal $\left(S_{\mathrm{P}}\right)$ at the nodes of a very fine square grid with a spacing of $100 \mathrm{~m}$ covering the study area. This grid is extended with 1000 randomly selected validation locations in between the grid-points.

In the next step the simulated signals at the grid-nodes and validation points are used to simulate fields with count data. One field with statistics closest to those of the SIBES data is selected, and underlying $S_{\mathrm{B}}$ and $S_{\mathrm{P}}$ fields are repeatedly sampled by grid-sampling. A range of grid-spacings is applied. Each selected grid is used to predict the model parameters $\pi_{i}$ and $\mu_{i}$ at the validation locations. By comparing these predictions with the true (original) model parameters at the validation points the quality of the predictions is assessed. More details on all steps but the first (estimation of ZIP model parameters) are given below. For 25 details on the first step we refer to Lyashevska et al. (2016).

1. The simulated signals $S_{\mathrm{B}}$ at the nodes of $100 \mathrm{~m}$ grid extended with 1000 validation locations are backtransformed using the inverse of the link function in Eq. 2 to give 100 fields of the prevalence parameter $\pi$ of the Bernoulli distribution. The same procedure is followed for the $S_{\mathrm{P}}$ to give 100 fields of the intensity parameter $\mu$ of the Poisson distribution. 
2. Apart from the field with zero-inflated counts, the two underlying fields with simulated prevalence parameter values $\pi$ and simulated intensity parameter values $\mu$ are selected, as these are needed in the validation. Fig. 1 shows a map of the product of $\pi$ and $\mu$, representing the unconditional intensity (unconditional expected count), and a map of the SIBES count data. There is a clear resemblance between the two maps.

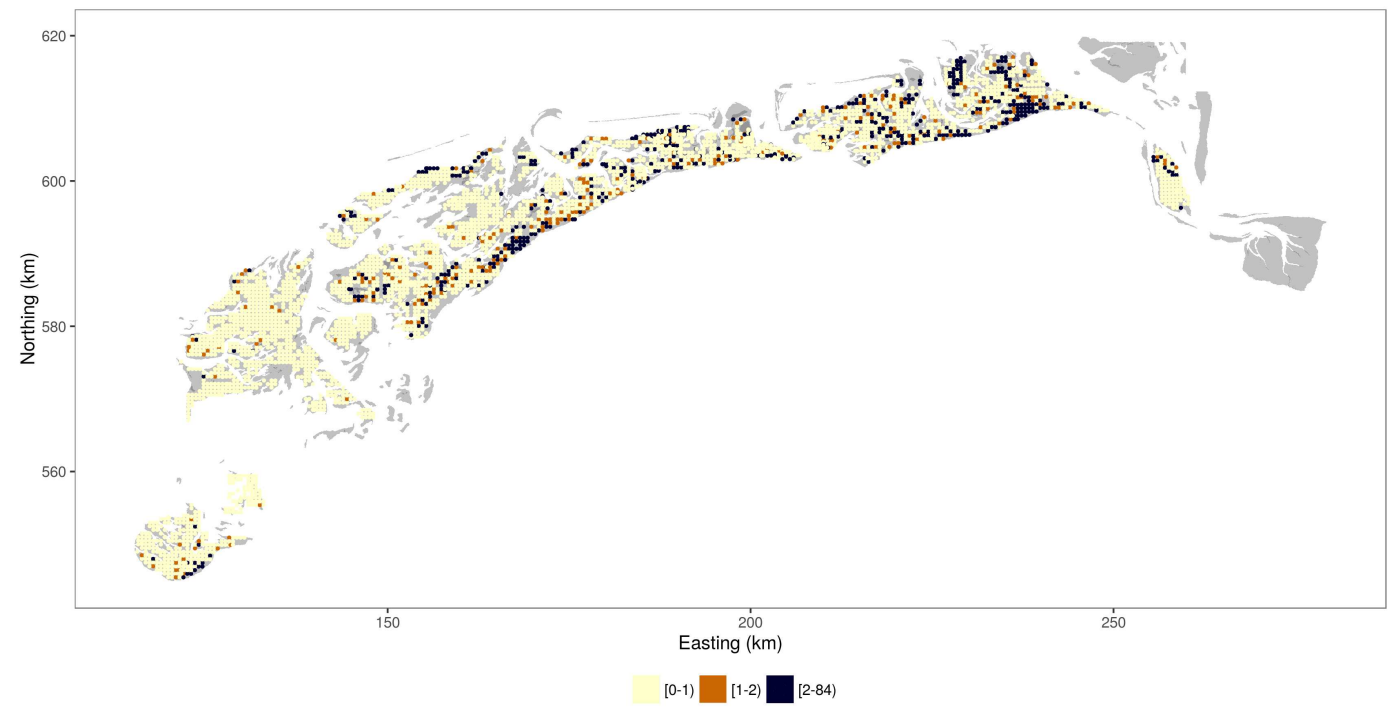

(a)

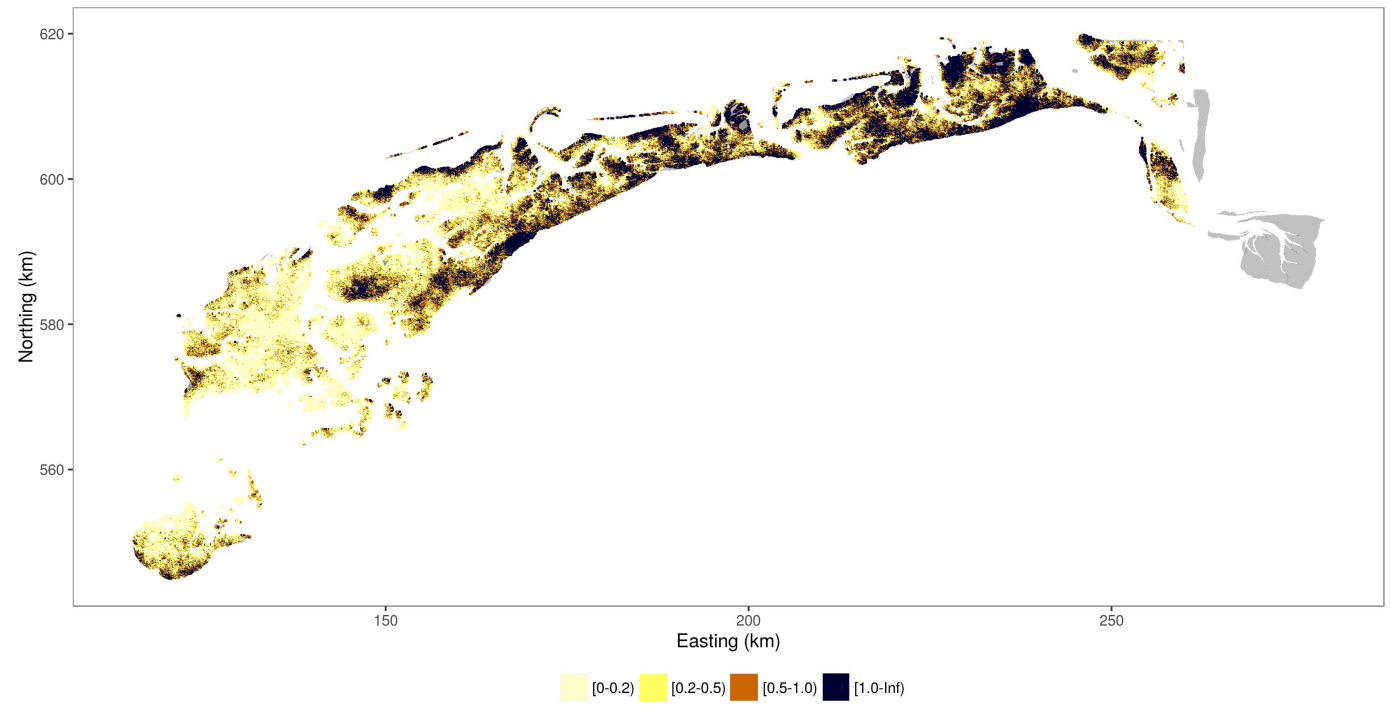

(b)

Figure 1: Empirical species abundance map of Macoma balthica (a) and unconditional intensity map (b) conditionally simulated to the nodes of $100 \mathrm{~m}$ grid.

3. The two underlying fields are sampled on a grid with a spacing of $200,400,800,1600$, and $3200 \mathrm{~m}$. For each grid-spacing 100 samples are randomly selected. The corresponding number of grid-points was on average $28505,7130,1783,446$, and 110, respectively. An overlay is made of the selected grids 
which are then used in prediction of the prevalence parameter $\pi$ and the intensity parameter $\mu$ at the validation locations.

\subsection{Quality measures}

The quality of the predicted prevalence parameters was quantified by the Mean Squared Error (MSE); for instance for the prevalence parameter $\pi$ this MSE equals :

$$
\operatorname{MSE}(\pi)=\frac{1}{n} \sum_{i=1}^{n}\left\{\hat{\pi}_{i}-\pi_{i}\right\}^{2}
$$

with $n$ the number of validation points $(n=1000), \hat{\pi}_{i}$ the predicted prevalence parameter at location $i$ and $\pi_{i}$ the 'pseudo-observed' intensity parameter. For intensity parameter $\mu$ MSE is computed from the subset of validation points with a simulated value of 1 for the presence/absence indicator (species present). This

intensity (intensity not conditioned on presence). For this product again all 1000 validation points are used. 
For each grid-spacing we have 100 grid-samples. All 100 grid-samples are used in prediction with the fixed parent-hyperparameters $\boldsymbol{\theta}_{\mathrm{B}}$ and $\boldsymbol{\theta}_{\mathrm{P}}$ (no sample-specific estimation), leading to 100 estimates of $\operatorname{MSE}(\boldsymbol{\pi})$, $\operatorname{MSE}(\mu)$ and $\operatorname{MSE}(\pi \cdot \mu)$. The distribution of these 100 estimates is an estimate of the sampling distribution samples are used in prediction with variable sample-specific estimates of the hyperparameters $\boldsymbol{\theta}_{\mathrm{B}}$ and $\boldsymbol{\theta}_{\mathrm{P}}$, so these five estimates give a very rough estimate of the sampling distribution only.

\section{Results}

\subsection{Prevalence}

for the prevalence parameter $\pi$. For the first four spacings the increase of the mean MSE was very modest, but beyond a spacing of $1600 \mathrm{~m}$, the increase was much stronger (Fig. 2C).

\section{Discussion and conclusions}

The aim of this paper was to quantify the effect of grid-spacing on the quality of spatial predictions of 195 the abundance of species that show zero-inflation and spatial autocorrelation. We proposed an approach in which multiple fields of the prevalance parameter $\pi$ and the intensity parameter $\mu$ of a zero-inflated Poisson mixture model are simulated by generalized linear geostatistical models. These fields were used to simulate pseudo-realities of zero-inflated autocorrelated counts. One pseudo-reality was then selected with summary 


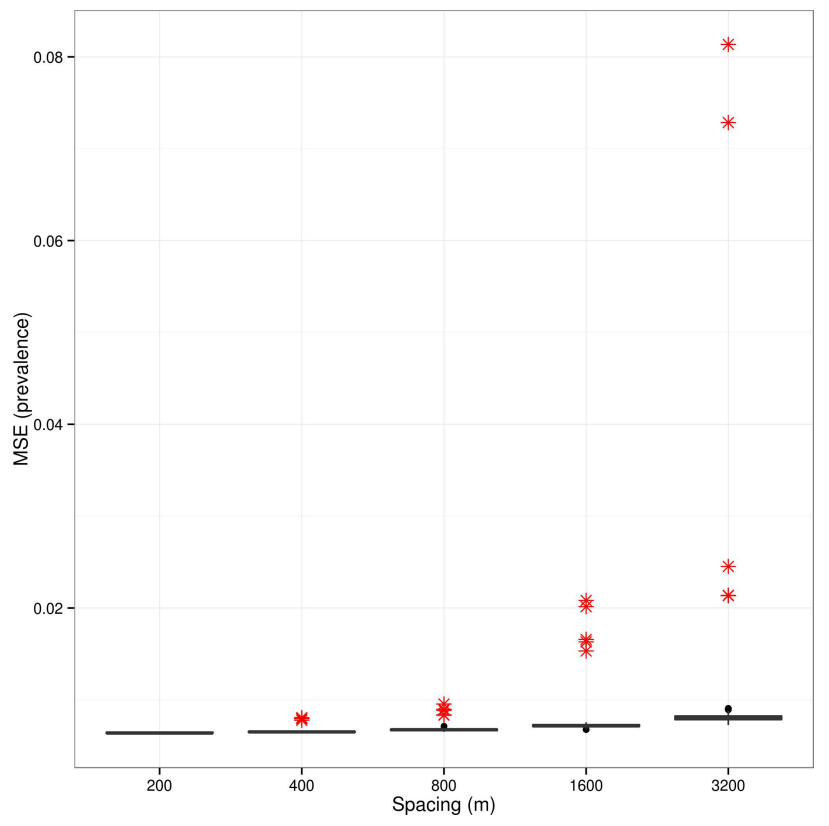

(a)

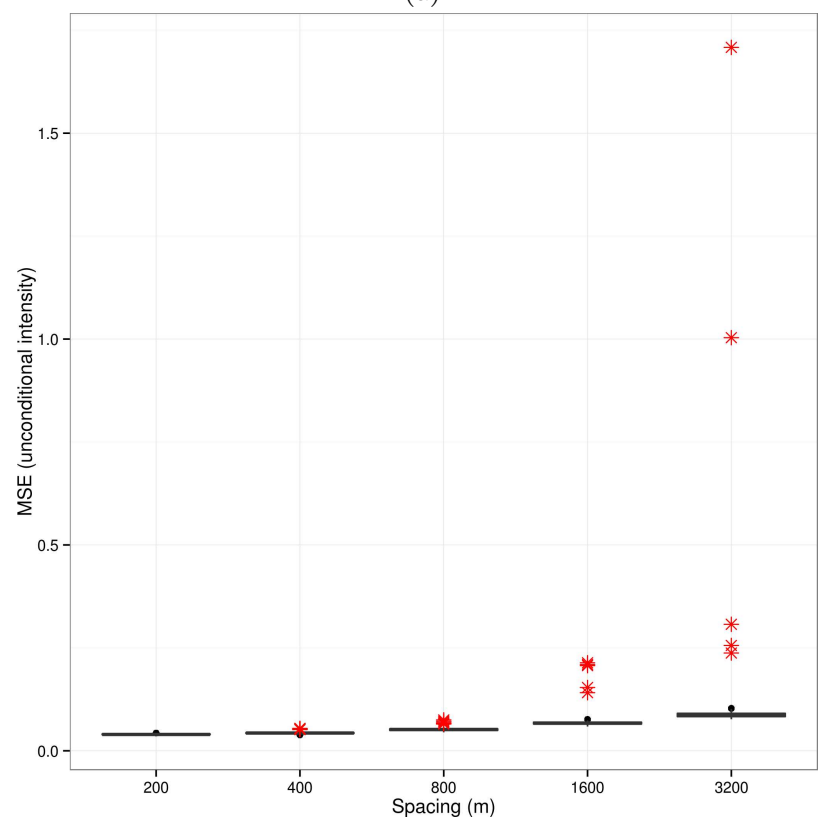

(c)

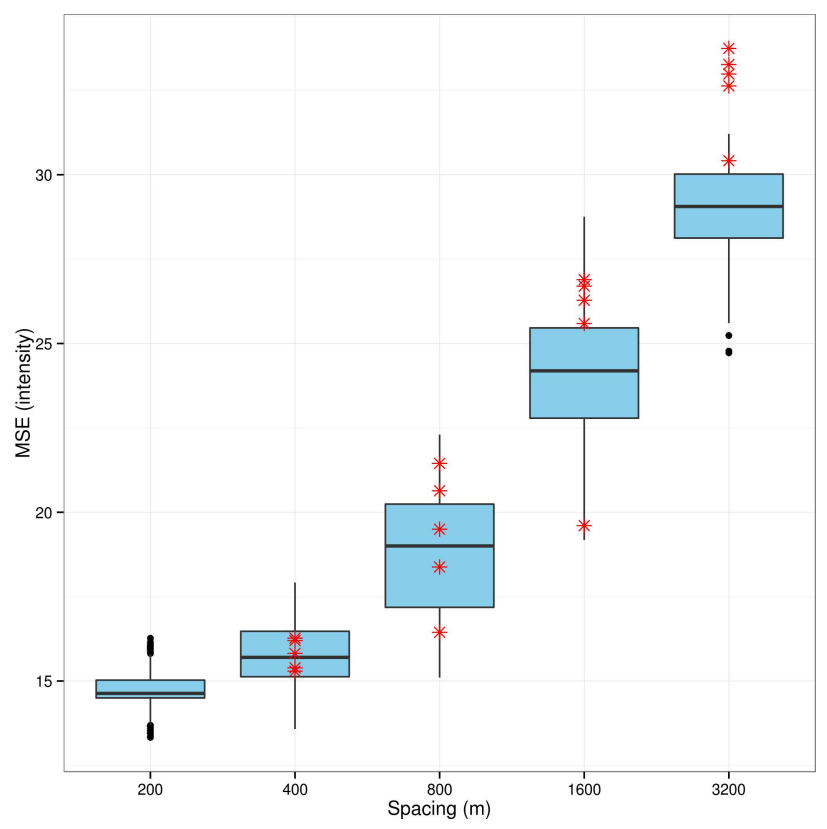

(b)

Figure 2: The MSE for predicted prevalence $(\pi)$ (a) predicted intensity $(\mu)$ (b) and predicted unconditional intensity $(\pi \cdot \mu)(\mathrm{c})$ as a function of grid-spacing for 200, 400, 800, 1600, and $3200 \mathrm{~m}$. Predictions were obtained by simple kriging with an external drift with fixed parent-hyperparameters (blue) and hyperparameters estimated from a sample (red). All 100 grid-samples were used in prediction with fixed parent-hyperparameters and only five grid-samples were used in prediction with variable sample-specific estimates. The first grid-spacing $(200 \mathrm{~m})$ with estimated hyperparameters could not be estimated due to memory constraints. 
statistics that were close to summary-statistics of the available data. This pseudo-reality was then sampled by the sampling design under study, in this study grid-sampling at various grid-spacings. A selected sample was used to predict at a fixed set of validation locations and to compute the MSE of the predictions of $\pi$ and $\mu$. By repeating the selection of samples and the prediction at the validation points, an estimate of the sampling distribution of MSE is obtained.

To construct the graph with the estimated sampling distribution of MSE against grid-spacing the hyper-

imation underestimates the contribution of the uncertainty about the hyperparameters to the uncertainty about the predictions, but it is a first attempt within reasonable computing time. To improve the quality of the estimated MSEs at various grid-spacings a more efficient procedure for estimating the hyperparameters is needed. We welcome research into estimation of the hyperparameters of a ZIP mixture model by integrated 
nested Laplacian approximation, as proposed by Rue et al. (2009).

For the time being we must base our decisions on the graphs as presented in Figs 2a, 2b] and 2c, The graphs of the prevalence parameter $\pi$ and of the unconditional intensity $\pi \cdot \mu$ show that beyond a spacing of $1600 \mathrm{~m}$ the mean of MSE starts increasing at a much faster rate. This is especially true for the MSEs obtained with hyperparameters estimated from the samples. Based on these findings the $1600 \mathrm{~m}$ grid which consists of 446 locations for our study area of $2400 \mathrm{~km}^{2}$ seems to be a good compromise between sampling costs and prediction accuracy. The increase in accuracy of the $800 \mathrm{~m}$ grid does not seem to outweigh the fourfold increase in number of sampling units taken. But of course the optimal spacing can only be determined when sampling costs and accuracy are defined in a common unit.

\section{Acknowledgements} supported by the Waddenfonds, and the provinces of Fryslân and Noord Holland (Grant/Award Number: WF209902). We thank the SIBES core-team, numerous volunteers, and the crew of the RV Navicula. In 2010 the SIBES-monitoring was carried out with financial support from NAM, NWO-ALW (ZKO programme) and Royal NIOZ.

\section{Supporting information}

Data and R-code can be found on https://github.com/lyashevska/GridSpacing-Paper.git

\section{References}

Agarwal, D., Gelfand, A., Citron-Pousty, S., 2002. Zero-inflated models with application to spatial count data. Environmental and Ecological Statistics 9, 341-355. URL: http://dx.doi.org/10.1023/A\%3A1020910605990, doi:10.1023/A:1020910605990.

Bijleveld, A.I., van Gils, J.A., van der Meer, J., Dekinga, A., Kraan, C., van der Veer, H.W., Piersma, T., 2012. Designing a benthic monitoring programme with multiple conflicting objectives. Methods in Ecology and Evolution 3, 526-536. URL: http://dx.doi.org/10.1111/j.2041-210X.2012.00192.x, doi $10.1111 /$ j.2041-210X.2012.00192.x.

Boyd, C., Woillez, M., Bertrand, S., Castillo, R., Bertrand, A., Punt, A.E., 2015. Bayesian posterior prediction of the patchy spatial distributions of small pelagic fish in regions of suitable habitat. Canadian Journal of Fisheries and Aquatic Sciences 72, 290-303.

Brus, D.J., Heuvelink, G.B.M., 2007. Optimization of sample patterns for universal kriging of environmental variables. Geoderma 138, 86-95. 
265 Caughlan, L., Oakley, K.L., 2001. Cost considerations for long-term ecological monitoring. Ecological Indicators 1, 123 - 134. URL: http://www.sciencedirect.com/science/article/pii/S1470160X01000152, doi http://dx.doi.org/10.1016/S1470-160X(01)00015-2

Clarke, K.R., Green, R.H., 1988. Statistical design and analysis for a 'biological effects' study. Marine Ecology Progress Series 46, 213-226.

270 Compton, T.J., Holthuijsen, S., Koolhaas, A., Dekinga, A., ten Horn, J., Smith, J., Galama, Y., Brugge, M., van der Wal, D., van der Meer, J., van der Veer, H.W., Piersma, T., 2013. Distinctly variable mudscapes: Distribution gradients of intertidal macrofauna across the Dutch Wadden Sea . Journal of Sea Research 82, 103 - 116. URL: http://www.sciencedirect.com/science/article/pii/S1385110113000300, doi http://dx.doi.org/10.1016/j.seares.2013.02.002 special issue: Proceedings of the International Symposium on the Ecology of the Wadden Sea.

Cozzi, G., Broekhuis, F., McNutt, J., Schmid, B., 2013. Density and habitat use of lions and spotted hyenas in northern botswana and the influence of survey and ecological variables on call-in survey estimation. Biodiversity and Conservation 22, 2937-2956. URL: http://dx.doi.org/10.1007/s10531-013-0564-7, doi $10.1007 / \mathrm{s} 10531-013-0564-7$.

Diggle, Peter J.and Ribeiro Jr., P.J., 2007. Model-based Geostatistics. Springer.

Fortin, M.J., Drapeau, P., Legendre, P., 1989. Spatial autocorrelation and sampling design in plant ecology. Vegetatio 83, pp. 209-222. URL: http://www.jstor.org/stable/20038496.

Foster, S.D., Hosack, G.R., Hill, N.A., Barrett, N.S., Lucieer, V.L., 2014. Choosing between strategies for designing surveys: autonomous underwater vehicles. Methods in Ecology and Evolution 5, 287-297. URL: http://dx.doi.org/10.1111/2041-210X.12156, doi:10.1111/2041-210X.12156.

van Groenigen, J., Siderius, W., Stein, A., 1999. Constrained optimisation of soil sampling for minimisation of the kriging variance. Geoderma 87, 239-259.

Guisan, A., Thuiller, W., 2005. Predicting species distribution: offering more than simple habitat models. Ecology Letters 8, 993-1009. URL: http://dx.doi.org/10.1111/j.1461-0248.2005.00792.x, doi $10.1111 / \mathrm{j} .1461-0248.2005 .00792 \cdot \mathrm{x}$.

Guisan, A., Zimmermann, N., 2000. Predictive habitat distribution models in ecology. Ecological Modelling $135,147-186$.

Hanberry, B., He, H., Dey, D., 2012. Sample sizes and model comparison metrics for species distribution models. Ecological Modelling 227, $29 \quad-$ 33. URL: http://www.sciencedirect.com/science/article/pii/S0304380011005837, doi http://dx.doi.org/10.1016/j.ecolmodel.2011.12.001. 
Hilbe, J., Greene, W., 2007. Count response regression models, in: Rao, C., Miller, J., Rao, D. (Eds.), Epidemiology and Medical Statistics. Elsevier. Elsevier Handbook of Statistics Series.

Lambert, D., 1992. Zero-Inflated Poisson Regression, with an Application to Defects in Manufacturing. Technometrics 34, pp. 1-14. URL: http://www.jstor.org/stable/1269547.

Legendre, P., Dale, M.R.T., Fortin, M.J., Gurevitch, J., Hohn, M., Myers, D., 2002. The consequences of spatial structure for the design and analysis of ecological field surveys. Ecography 25, pp. 601-615.

Lewis, F., Butler, A., Gilbert, L., 2011. A unified approach to model selection using the likelihood ratio test. Methods in Ecology and Evolution 2, 155-162. URL: http://dx.doi.org/10.1111/j.2041-210X.2010.00063.x doi 10.1111/j.2041-210X.2010.00063.x.

Lyashevska, O., Brus, D.J., van der Meer, J., 2016. Mapping species abundance by a spatial zeroinflated Poisson model: a case study in the Wadden Sea, the Netherlands. Ecology and Evolution URL: http://dx.doi.org/10.1002/ece3.1880, doi:10.1002/ece3.1880.

Martin, T.G., Wintle, B.A., Rhodes, J.R., Kuhnert, P.M., Field, S.A., Low-Choy, S.J., Tyre, A.J., Possingham, H.P., 2005. Zero tolerance ecology: improving ecological inference by modelling the source of zero observations. Ecology Letters 8, 1235-1246. URL: http://dx.doi.org/10.1111/j.1461-0248.2005.00826.x doi 10.1111/j.1461-0248.2005.00826.x.

McBratney, A., Webster, R., 1981. The design of optimal sampling schemes for local estimation and mapping of regionalized variables-ii. program and examples. Computers and Geosciences 7, 335-365. Cited By (since 1996) 53.

Pearson, R.G., Raxworthy, C.J., Nakamura, M., Townsend Peterson, A., 2007. Predicting species distributions from small numbers of occurrence records: a test case using cryptic geckos in madagascar. Journal of Biogeography 34, 102-117. URL: http://dx.doi.org/10.1111/j.1365-2699.2006.01594.x, doi $10.1111 / j .1365-2699.2006 .01594 . x$.

Perner, J., Schueler, S., 2004. Estimating the density of ground-dwelling arthropods with pitfall traps using a nested-cross array. Journal of Animal Ecology 73, pp. 469-477. URL: http://www.jstor.org/stable/3505657.

Rachowicz, L.J., Hubbard, A.E., Beissinger, S.R., 2006. Evaluating at-sea sampling designs for marbled murrelets using a spatially explicit model. Ecological Modelling 196, 325329 - 344. URL: http://www.sciencedirect.com/science/article/pii/S0304380006000731, doi http://dx.doi.org/10.1016/j.ecolmodel.2006.02.011.

Recta, V., Haran, M., Rosenberger, J.L., 2012. A two-stage model for incidence and prevalence in point-level spatial count data. Environmetrics 23, 162-174. URL: http://dx.doi.org/10.1002/env.1129. 
Reynolds, J.H., Thompson, W.L., Russell, B., 2011. Planning for success: Identifying effective and efficient survey designs for monitoring. Biological Conservation 144, 1278 1284. URL: http://www.sciencedirect.com/science/article/pii/S0006320710004970, doi http://dx.doi.org/10.1016/j.biocon.2010.12.002

Rue, H., Martino, S., Chopin, N., 2009. Approximate bayesian inference for latent gaussian models by using integrated nested laplace approximations. Journal of the Royal Statistical Society: Series B (Statistical Methodology) 71, 319-392. URL: http://dx.doi.org/10.1111/j.1467-9868.2008.00700.x.

Stockwell, D.R., Peterson, A., 2002. Effects of sample size on accuracy of species distribution models. Ecological Modelling 148, 1 - 13 . URL: $\quad$ http://www.sciencedirect.com/science/article/pii/S030438000100388X, doi http://dx.doi.org/10.1016/S0304-3800(01)00388-X.

Tyre, A.J., Tenhumberg, B., Field, S.A., Niejalke, D., Parris, K., Possingham, H.P., 2003. Improving precision and reducing bias in biological surveys: estimating false-negative error rates. Ecological Applications 13, $1790-1801$.

Vieira, C., Seneca, A., Sergio, C., 2012. Floristic and ecological survey of bryophytes from portuguese watercourses. Cryptogamie, Bryologie 33, 113-134.

Webster, R., Oliver, M.A., 2007. Geostatistics for environmental Scientists. 2 ed., John Wiley.

Wisz, M.S., Hijmans, R.J., Li, J., Peterson, A.T., Graham, C.H., Guisan, A., NCEAS Predicting Species Distributions Working Group, 2008. Effects of sample size on the performance of species distribution models. Diversity and Distributions 14, 763-773. URL: http://dx.doi.org/10.1111/j.1472-4642.2008.00482.x doi $10.1111 / j .1472-4642.2008 .00482 . x$.

Zurell, D., Berger, U., Cabral, J.S., Jeltsch, F., Meynard, C.N., Münkemüller, T., Nehrbass, N., Pagel, J., Reineking, B., Schröder, B., Grimm, V., 2010. The virtual ecologist approach: simulating data and observers. Oikos 119, 622-635. URL: http://dx.doi.org/10.1111/j.1600-0706.2009.18284.x, doi $10.1111 / \mathrm{j} .1600-0706.2009 .18284 . \mathrm{x}$. 